\title{
Comparison of the Middle and Lower Serratus Anterior Muscle Activities during Various Wall Push-up Plus Exercises
}

\author{
WON-GYU Yoo ${ }^{1)}$ \\ 1) Department of Physical Therapy, College of Biomedical Science and Engineering, Inje University \\ and Elderly Life Redesign Institute: 607 Obangdong, Gimhae, Gyeongsangnam-do 621-749, Republic \\ of Korea.TEL: +82 55-320-3994,FAX:+82: 55-329-1678,E-mail:won7y@inje.ac.kr
}

\begin{abstract}
Purpose] This study compared the effects of wall push-up plus conditions on activation of the middle serratus anterior (MSA) and lower serratus anterior (LSA), and investigated which exercise selectively activated each part of the serratus anterior. [Subjects] Sixteen subjects were recruited. [Methods] Surface electrodes were placed over the middle and lower serratus anterior. The subjects performed the wall push-up plus exercise under three conditions. [Result] Condition $3(39.9 \pm 12.4 \%)$ significantly increased MSA activity compared to condition $1(32.0 \pm 16.8 \%)$. Condition $2(40.7 \pm 15.2 \%)$ significantly increased LSA activity compared to condition 1 $(32.6 \pm 10.5 \%)$. [Conclusion] We think that each part of the serratus anterior muscle is activated differently according to the protraction direction and surface condition during wall push-up plus exercise.

Key words: Electromyography, Serratus anterior, Wall push-up exercise
\end{abstract}

(This article was submitted Aug. 15, 2012, and was accepted Sep. 14, 2012)

\section{INTRODUCTION}

Scapular setting exercises are used to stabilize the shoulder complex. Closed kinetic-chain exercises, such as a standard push up or push up plus, have been shown to stimulate mechanoreceptors that contribute to shoulder joint stabilization $^{1,2)}$. Weakness of the serratus anterior causes poor scapular orientation and instability, thereby conributing to pathologic kinematics such as impingement ${ }^{3}$. The push up plus exercise is used to induce scapular elevation, and upward rotation needs to have minimal effect on the upper trapezius and selective stimulation of the serratus anterior and lower trapezius ${ }^{4)}$. The serratus anterior has many branches and can be divided into the upper, middle, and lower parts ${ }^{5,6)}$. Some researchers have shown that the middle and lower parts of serratus anterior muscle are important for stabilizing scapular movement, and that activating the middle and lower part of the serratus anterior muscle might be an effective form of intervention ${ }^{7,8)}$. Wall push-ups can be used as a low-level strengthening exercise for shoulder muscle rehabilitation and can prepare the shoulder muscles before advancing to floor push-ups. Wall push-ups are also a safe exercise for sports players after shoulder surgery. Therefore, the purpose of the present study was to compare the effects of wall push-up plus conditions on the selective activation of the middle and lower serratus anterior.

\section{SUBJECTS AND METHODS}

This study was performed with sixteen males aged 20-29 years $(26 \pm 4.0 \mathrm{yrs})$, with a weight of $65 \pm 6.3 \mathrm{~kg}$ and height of $176 \pm 8.2 \mathrm{~cm}$ as subjects. The subjects had no history of traumatic injury or discomfort in their upper extremities during the past 6 months. Electromyography (EMG) signals were amplified, and sampled at $1000 \mathrm{~Hz}$ using a MP 150 system (Biopack System, Santa Barbara, CA, USA). The surface electrodes were placed on the dominant right side of the middle serratus anterior (MSA), at approximately the mid-axillary line of the right side over the 5th rib, and the lower serratus anterior (LSA), on the belly of the muscle that branched to the 7 th rib $^{9,10)}$. EMGs of maximal voluntary isometric contractions were recorded to enable normalization of the EMG amplitude. The subjects performed wall push-up plus exercises in a standing position under 3 conditions: condition 1 was a stable wall push-up plus, with the shoulder at 90 degrees of flexion; condition 2 was a stable wall push-up plus, with the shoulder at 120 degrees flexion; and condition 3 was a labile wall push-up plus exercise with the shoulder at 90 degrees flexion. An air cushion ball (15 inches) was used as the labile wall surface. Elbow flexion during the wall push-up plus was restricted according to the examiner's instructions. The SPSS statistical package (version 12.0; SPSS, Chicago, IL, USA) was used for statistical analysis, and the statistical significance of differences was examined by repeated one-way ANOVA, with significance accepted at value of $\mathrm{p}<0.05$.

\section{RESULTS}

Condition 3 (39.9 $\pm 12.4 \%)$ significantly increased MSA 
activity compared to condition $1(32.0 \pm 16.8 \%)(\mathrm{p}<0.05)$ MSA activity in condition 2 was $33.9 \pm 20.5 \%$. Condition $2(40.7 \pm 15.2 \%)$ significantly increased LSA activity compared to condition $1(32.6 \pm 10.5 \%)(\mathrm{p}<0.05)$. LSA activity in condition 3 was $34.2 \pm 19.8 \%$.

\section{DISCUSSION}

Push-up plus exercise involves posterior translation of the thorax on relatively fixed scapulae, which can be done alone or in combination with push-ups ${ }^{11)}$. Ludewig et al. ${ }^{4}$ ) suggested that the serratus anterior was activated to a greater extent in push-up plus than in standard push-ups. The present study are performed to evaluate whether the middle and lower parts of the serratus anterior muscle were activated differently during wall push-up plus exercises under different conditions. Condition 2, with 120 degree shoulder flexion, significantly increased LSA activity compared to condition 1 (90 degree shoulder flexion). Ekstrom et al. ${ }^{12)}$ reported that diagonal arm elevation and arm elevation above $120^{\circ}$ activated the lower serratus anterior more than other exercises. In our view, LSA was more highly activated when the scapula was protracted by the wall push-up plus with arm elevation of $120^{\circ}$ than with arm elevation of $90^{\circ}$. Condition 3 using a labile wall surface significantly increased MSA activity compared to stable wall surface push-up plus. The results of the present study show that MSA exhibited greater activity in stabilizing the scapular position when a labile wall surface was used than when a stable wall surface was used. Of the scapulothoracic musculature that attaches to the scapula, the trapezius and serratus anterior provide a stabilizing effect on the inferior angle of the scapula with a couple-forced mechanism ${ }^{7)}$. We consider that MSA was activated in movement requiring static stability or balance of the scapulothoracic joint. The activation of specific parts of the serratus anterior is difficult in clinical practice, and this study identified selective wall push-up exercise methods for the middle and lower serratus anterior muscles using different conditions.

\section{ACKNOWLEDGEMENTS}

This research was supported by Basic Science Research Program through the National Research Foundation of Korea (NRF) funded by the Ministry of Education, Science and Technology (No. 2012001058).

\section{REFERENCES}

1) Liebenson C: Self-management of shoulder disorder-part3: treatment self help patient's advice. J Bodyw Mov Ther, 2006, 10: 65-70. [CrossRef]

2) Lephart SM, Henry TJ: The physiological basis for open and closed kinetic chain rehabilitation for the upper extremity. J Sport Rehabil, 1996, 5: 71-87.

3) Mottram SL: Dynamic stability of the scapula. Man Ther, 1997, 2: 123-131. [Medline] [CrossRef]

4) Ludewig PM, Hoff MS, Osowski EE, et al: Relative balance of serratus anterior and upper trapezius muscle activity during push-up exercises. Am J Sports Med, 2004, 32: 484-493. [Medline] [CrossRef]

5) Ekstrom RA, Bifulco KM, Lopau CJ, et al.: Comparing the function of the upper and lower parts of the serratus anterior muscle using surface electromyography. J Orthop Sports Phys Ther, 2004, 34: 235-243. [Medline]

6) Martin RM, Fish DE: Scapular winging: anatomical review, diagnosis, and treatments. Curr Rev Musculoskelet Med, 2008, 1: 1-11. [Medline] [CrossRef]

7) Inman VT, Saunders JB, Abbott LC: Observations on the function of the shoulder joint. J Bone Joint Surg, 1944, 26: 1-31.

8) Park SY, Yoo WG: Differential activation of parts of the serratus anterio muscle during push-up variations on stable and unstable bases of support. J Electromyogr Kinesiol, 2011, 21: 861-867. [Medline] [CrossRef]

9) Lehman GJ, Gilas D, Patel U: An unstable support surface does not increase scapulothoracic stabilizing muscle ctivity during push up and push up plus exercises. Man Ther, 2008, 13: 500-506. [Medline] [CrossRef]

10) Holtermann A, Mork PJ, Andersen LL, et al.: The use of EMG biofeedback for learning of selective activation of intra-muscular parts within the serratus anterior muscle: a novel approach for rehabilitation of scapular muscle imbalance. J Electromyogr Kinesiol, 2010, 20: 359-365. [Medline] [CrossRef]

11) Lunden JB, Braman JP, Laprade RF, et al.: Shoulder kinematics during the wall push-up plus exercise. J Shoulder Elbow Surg, 2010, 19: 216-223. [Medline] [CrossRef]

12) Ekstrom RA, Soderberg GL, Donatelli RA: Normalization procedure using maximum voluntary isometric contractions for the serratus anterior and trapezius muscles during surface EMG analysis. J Electromyogr Kinesiol, 2005, 15: 418-428. [Medline] [CrossRef] 